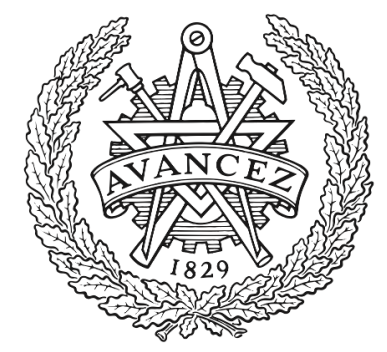

CHALMERS

UNIVERSITY OF TECHNOLOGY

\title{
Using a Superconducting Gravimeter in Support of Absolute Gravity Campaigning — A Feasibility Study
}

Downloaded from: https://research.chalmers.se, 2023-04-26 08:41 UTC

Citation for the original published paper (version of record):

Scherneck, H., Rajner, M. (2019). Using a Superconducting Gravimeter in Support of Absolute Gravity Campaigning - A Feasibility

Study. Geophysica, 54(1): 117-135

N.B. When citing this work, cite the original published paper. 
Geophysica (2019), 54(1), 117-135

\title{
Using a Superconducting Gravimeter in Support of Absolute Gravity Campaigning — A Feasibility Study
}

\author{
Hans-Georg Scherneck and Marcin Rajner \\ Onsala Space Observatory, Department of Earth, Space and Environment, \\ Chalmers University of Technology, Sweden
}

(Submitted: October 31, 2018; Accepted: February 28, 2019)

\begin{abstract}
Preparing for joint analysis of absolute gravity (AG) campaigns, this report investigates whether a stationary superconducting gravimeter (SCG) can provide a long-term stable measurement of sitedependent perturbations that help in reduction to the local value of little-g and its secular rate of change. The crucial element concerns the discrimination of instrumental drift components from trends of physical origin, where biasses in the inferred long-term drift rate may offset the rate that the reduced AG campaigns deliver. Thus, the main objective is to include a set of gravity models and proxy series as complete as possible in the SCG analysis. Findings indicate consistency for $\dot{g}$ in the drift model at the $0.5 \mathrm{~nm} / \mathrm{s}^{2} / \mathrm{yr}$ level using observations at Onsala Space Observatory from 2009 to 2017. In pursuit of the overriding objective to improve the accuracy of secular rates of gravity owed to Glacial Isostatic Adjustment, our approach may even put numbers on a range of long-term changes due to atmosphere, hydrology, and non-tidal ocean loading, namely the rate biasses reported here.
\end{abstract}

Keywords: Gravity change, Glacial Isostatic Rebound, Superconducting Gravimeter, absolute gravimeter

\section{$1 \quad$ Introduction}

The European North exhibits the phenomenon of uplift encompassing the whole of Fennoscandia as a late and long-lasting consequence of the recession of the Pleistocene ice sheet with a maximum of glaciation 25,000 to 18,000 years b.p. and a major melt-down commencing 16,500 years b.p. (Lambeck et al., 2014). The ensuing process of Glacial Isostatic Rebound (GIA) comprises gravity change, an effect to which efforts of precise measurement have been dedicated since 1966 (Ekman and Mäkinen, 1996), until the early $2000 \mathrm{~s}$ with spring gravimeters along profiles, and thereafter preferably with modern absolute gravimeters, the Micro-g FG5 model as the flagship. Yet, the gravity rate of change appears much harder to determine at the desired precision than its kinematic counterparts, vertical and horizontal deformation rates. Plenty of studies using relative sea level change and GNSS-observations have been able to bracket ranges for lithosphere thickness and mantle viscosity (e.g. Milne et al., 2004). Gravity would afford us a complementary resource for inversion of observations to resolve earth interior structure and dynamics. The first order effect in gravity is related to the vertical movement of a gravimeter in the gradient, which is dominated by the free-air decrease of $g$, and countered by an integrated attraction of mantle mass migrating in under the 
uplifting dome. It would be desirable to discern the competent layers and harness their fingerprints owed to their respective densities. These imprints don't even come as a second order effect. Greater yet is the impact of the size of the former ice sheet, which has more to do with the spherical geometry and the flexibility of the lithosphere under loads of different wavelength. These circumstances have been scrutinized by Olsson et al. (2012, 2015). Given the limited precision of absolute gravimeters (AG), say, $\pm 20 \mathrm{~nm} / \mathrm{s}^{2}$, arriving at rate resolution of $\dot{g}$ of $0.4 \mathrm{~nm} / \mathrm{s}^{2} / \mathrm{yr}$ would require 30 years of annual remeasurement.

It might seem doubtful whether a stationary gravimeter (SG) could be of much help to eliminate systematic biases in long-term results from repeated AG campaigns. Such biases are intended to be characterized and mitigated in the international comparison meetings, second thoughts like those of Olsson et al. (2016) notwithstanding, and by remeasurement of stations' gravity gradients. Yet, an SG can help to increase the precision of the AG reduction model's tides and atmosphere parameters, assuming stationarity in that part, and that the repeats would randomise the perturbations, but also would resample at similar seasonal conditions (call it constructive aliasing). On the short time scale, e.g. during the few days of an AG campaign, an SG can provide realtime information (lest an offset and a scale factor need to be determined) replacing the time-dependent part of the reduction model. However, on a multi-year time scale, the mean value against which these gravity perturbations are specified, must be stable to at least the precision of the AG rate uncertainty aimed at. Such feat has rarely been attempted yet, at least not in the realm of GIA where persistent, constant rates of change are prospected.

Using a superconducting gravimeter ( $\mathrm{SCG}$, no. 054) in continuous operation, sampling at an interval of one second for now nine years, we will demonstrate that instrumental effects can be identified and isolated at the $0.4 \mathrm{~nm} / \mathrm{s}^{2} / \mathrm{yr}$ level in terms of rate uncertainty. In the strategy laid out, a joint analysis of all AG campaigns' drop measurements in one adjustment, we strive to avoid a bias in AG inferred rates incurred from residual SCG instrumental effects. This pursuit has been inspired by the work of Wziontek et al. (2009).

\section{GIA observation capabilities at Onsala}

Onsala Space Observatory (OSO) is equipped with a range of observing capabilities; we mention here the SWEPOS stations ONS* using GNSS and the VLBI activities concentrating on what they've been delivering in terms of vertical motion. From 2016 on, OSO has a precision mareograph; however, to estimate the sea-level rate we need a longer history, so the tide gauges at Ringhals and Göteborg-Torshamnen operated by Swedish Meteorological and Hydrological Institute (SMHI) were used. We compare the observations with model results in Table 1.

Since June 2012 Uppsala University operates a three-component broadband seismometer at OSO within their SNSN network (station ONA). The Guralp CMG-3t in- 
strument has its long-period band edge at $120 \mathrm{~s}$. It is installed $800 \mathrm{~m}$ west of the gravimetry lab.

Table 1. Rates of uplift and gravity change at Onsala Space Observatory.

\begin{tabular}{|c|c|c|c|c|}
\hline \multicolumn{2}{|c|}{ Model or observation } & rate & stddev & stddev \\
\hline \multicolumn{5}{|l|}{ Vertical rates $[\mathrm{mm} / \mathrm{yr}]$} \\
\hline GNSS JPL & 2009-2018 & 2.778 & $0.121^{a}$ & $0.0532^{b}$ \\
\hline GNSS M. Rajner, Gipsy & $2009-2018$ & 2.667 & $0.121^{a}$ & $0.0522^{b}$ \\
\hline \multicolumn{2}{|l|}{ GNSS ITRF2014 } & 2.83 & 0.05 & \\
\hline \multicolumn{2}{|l|}{ VLBI ITRF2014 } & 2.82 & 0.06 & \\
\hline \multicolumn{2}{|l|}{ GIA model $120-0.5-5^{c}$} & 2.38 & & \\
\hline \multicolumn{2}{|l|}{ GIA model $120-0.8-10^{c}$} & 2.26 & & \\
\hline \multicolumn{2}{|l|}{ GIA model $120-1.0-20^{c}$} & 1.70 & & \\
\hline \multirow[t]{4}{*}{ Rel. sea level } & Ringhals 1968-2016 & 0.3 & 0.6 & \\
\hline & Göteborg 1968-2018 & 1.6 & 0.4 & \\
\hline & Göteborg 1968-2018 ${ }^{d}$ & 1.4 & 0.3 & \\
\hline & Göteborg 1968-2018 $8^{d, e}$ & 0.7 & 0.3 & \\
\hline \multicolumn{5}{|l|}{ Gravity $\left[\mathrm{nm} / \mathrm{s}^{2} / \mathrm{yr}\right]$} \\
\hline \multirow[t]{2}{*}{ Model 120-0.5-10 } & (Olsson et al., 2015) & -3.56 & 0.12 & \\
\hline & (Olsson et al., 2019) & $-4.7,-3.0$ & 1.1, & 0.7 \\
\hline $\mathrm{AG}$ & 7-yr OSO campaigns, SCG-backed & t.b.d. & $0.13-0.2$ & \\
\hline $\mathrm{AG}$ & $(\text { Olsson et al., 2016) })^{g}$ & $-4.5,-3.6$ & $>5$ & \\
\hline \multirow{2}{*}{$\begin{array}{l}\mathrm{AG} \\
\mathrm{AG}\end{array}$} & (Timmen et al., 2015) $^{g}$ & -1.9 & 1.5 & \\
\hline & $\left(\right.$ Timmen et al., 2015) ${ }^{h}$ & -2.2 & 1.7 & \\
\hline \multicolumn{2}{|c|}{${ }^{a}$ flicker noise, Monte-Carlo } & ended Gauss & arkov (PEF & \\
\hline \multicolumn{5}{|c|}{${ }^{c}$ numbers of model mean lithosphere thickness $(\mathrm{km})$, upper and lower mantle viscosity $\left(10^{21} \mathrm{~Pa} \mathrm{~s}\right)$ respectively } \\
\hline \multicolumn{2}{|l|}{${ }^{d}$ air pressure added } & and Monte & rlo & \\
\hline \multicolumn{2}{|c|}{${ }^{f}$ 2010-2015, FG5-233, analysis variants (a) resp. (d) } & 3-2014, all & & \\
\hline \multicolumn{2}{|l|}{${ }^{h}$ 2003-2014, FG5-220 } & & & \\
\hline
\end{tabular}

\section{$3 \quad$ Superconducting gravimetry}

This section assumes SCG's of the GWR brand (Goodkind and Warburton, 1975). We denote drift as the instrumental contributions to the gravity readings. It typically consists of an initial exponential with a decay time of some hundred hours following the installation and a linear function of time. Offsets and new initiations of linear rates and exponentials may occur in response to service work on the instrument, but then at wellconstrained instances of time; however, unexpected jumps have been reported (Bützler, 2018). 
To begin with, the readings of the SCG are in units of Volt, and the scale factor needs determination. A widely used method employs AG's for the task, driven to high perfection in Van Camp et al. (2015) and Crossley et al. (2018).

\subsection{Gravimetry at Onsala}

The AG campaigns at Onsala are listed in Table 2. To introduce the vocabulary used in the sequel we use campaign for an instrument visit during which a number of setups, alternately called projects take place when the AG measures on different monuments and in different orientations. As the basis of the g-value to be determined the AG performs drops of the test mass typically every five seconds and 50 times in a row, then pausing for a number of minutes. The drops collected in between the pauses are combined to sets.

Table 2. Absolute Gravity campaigns at Onsala Space Observatory. The campaign identifier shows year and month. Offsets for the AGs are taken from the international comparison campaigns. Weighted mean and RMS are given in $\mathrm{nm} / \mathrm{s}^{2}$.

\begin{tabular}{rrrrrr}
\hline No. & Identifier & no. of drops & instrument & w.mean & w.rms \\
\hline 1 & $200907 \mathrm{a}$ & 8,704 & FG5-233 & 13.3 & 94.5 \\
2 & $200911 \mathrm{a}$ & 7,776 & FG5-220 & -12.4 & 140.1 \\
3 & $201004 \mathrm{a}$ & 5,462 & FG5-220 & 15.7 & 135.6 \\
4 & $201006 \mathrm{a}$ & 6,174 & FG5-233 & -3.6 & 54.6 \\
5 & $201009 \mathrm{a}$ & 2,623 & FG5-233 & -4.5 & 82.5 \\
6 & $201106 \mathrm{a}$ & 4,780 & FG5-233 & -12.2 & 55.8 \\
7 & $201106 \mathrm{~b}$ & 14,193 & FG5-220 & 0.0 & 124.3 \\
8 & $201106 \mathrm{c}$ & 2,399 & FG5-220 & -16.0 & 130.5 \\
9 & $201304 \mathrm{a}$ & 16,751 & FG5-233 & 9.1 & 92.0 \\
10 & $201405 \mathrm{a}$ & 25,630 & FG5-233 & -1.9 & 52.5 \\
11 & $201405 \mathrm{~b}$ & 4,591 & FG5-220 & 3.3 & 100.0 \\
12 & $201502 \mathrm{~b}$ & 10,470 & FG5-220 & 24.1 & $168.0^{b}$ \\
13 & $201502 \mathrm{~g}$ & $29,245^{a}$ & GAIN & n.a. & 35.8 \\
14 & $201502 \mathrm{~h}$ & $42,549^{a}$ & GAIN & n.a. & 17.0 \\
15 & $201505 \mathrm{a}$ & 28,114 & FG5-233 & 6.9 & 70.0 \\
16 & $201606 \mathrm{a}$ & 52,647 & FG5-233 & -7.0 & 52.4 \\
17 & $201707 \mathrm{a}$ & 7,910 & FG5-233 & -14.1 & 55.9 \\
\hline
\end{tabular}

${ }^{a}$ number of samples at 10 or $12 \mathrm{~s}$ intervals

${ }^{b}$ unreduced for microseismic noise

Throughout this work we use AG data at the drop level. In campaigns with SCG calibration as main purpose this data type, unreduced except for a constant, is the one to be used, and experience from experiments with order of 40,000 drops has been published in e.g. Meurers (2012). His work is particularly interesting here since it considers drift on the part of the AG as a potential source of bias. In order to avoid the variance bias due to microseismic accelerations present in the SCG records (Van Camp et al., 
2015), the time series is low-pass filtered. At that stage, the delay incurred by the SCG's anti-aliasing filter (the so-called GGP filter) is compensated by inverse application of its spectrum (the low-pass filtering is carried out in the Fourier domain reduced to a Nyquist frequency of $0.05 \mathrm{~Hz}$, thereupon the original sampling is restored with Fourier interpolation). Thus, the SCG-record is precisely timed by its GPS clock; however, the weak part of timing is found in the AG, where the drops are time-stamped according to the clock in the supervising computer, which can be off by several seconds. This clock drift can act adversely on mitigation of the AG's drop noise in the presence of strong microseisms, situations in which we can show efficient reduction to normal drop noise using a seismometer. Here, timing stability is crucial at the 1-second level, see Figures 1 and 2. (Since 2017 OSO relays the Internet time service safely via an internal Ethernet connection inside the lab.)

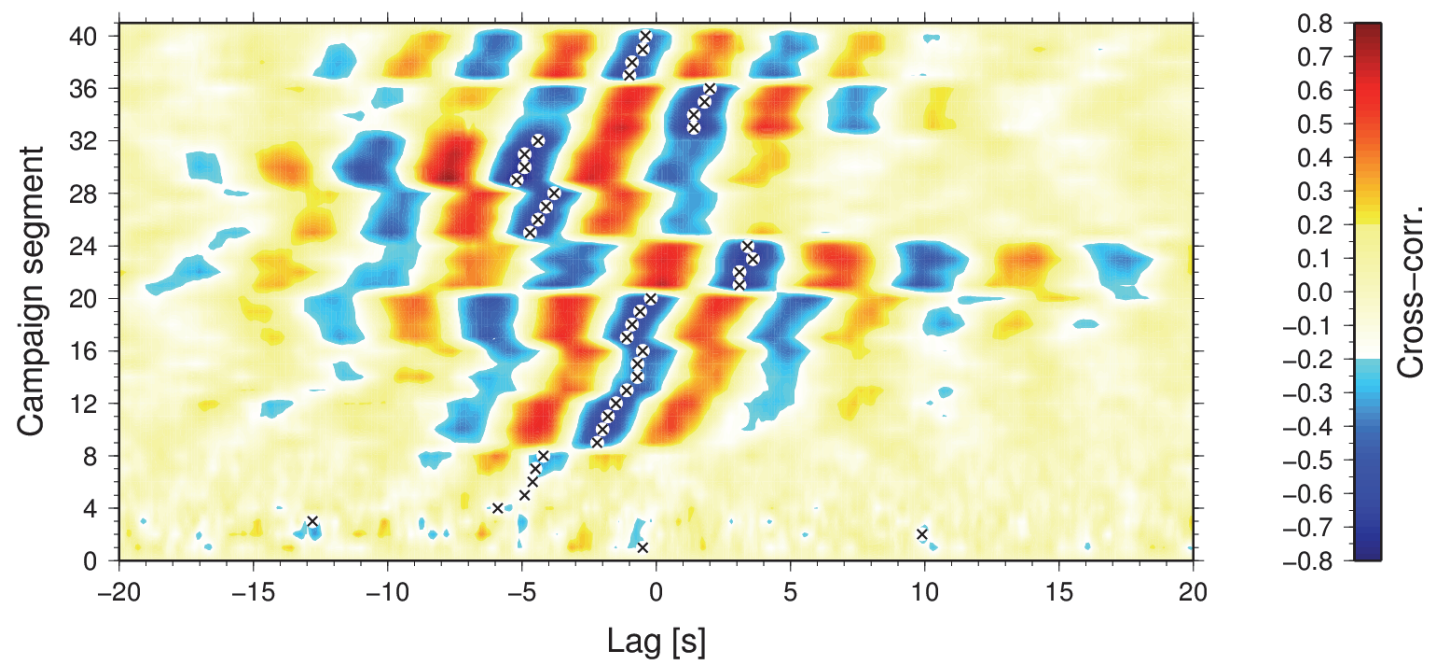

Fig. 1. Cross correlation of FG5-220 drop derivations at $10 \mathrm{~s}$ interval with acceleration at 10 samples per second derived from the SNSN-ONA broadband seismometer. The pattern shows peak correlation with a negative sign (emphasized by small crosses), suggesting that the AG's superspring over-compensates ground acceleration. Had the time stamping held a steady pace would the pattern show a strictly vertical striping. However, drifts to excursions up to four seconds can be seen. The diagram was prepared for the uniquely high microseismic activity during the campaign in Feb. 2015.

A scale factor $S$ for the SCG can be estimated separately in each campaign, $S_{c}$, basically following the method first introduced by Francis (1997) and Francis et al. (1998); however, in our adaptation we use AG drop data. The SCG voltage is synchronously picked after the conditioning described before. In Fig. 3 the factors are shown together with results from a range of alternative methods. As the multi-campaign analysis shall suggest, the AGs might be affected by drifts during some setups. Meurers (2012) results, testing polynomial degrees up to 10, exhibit the major gain in reduction already with straight-lines; therefore, slopes were estimated also in one of the singlecampaign variants. In both cases the $S_{c}$ 's are scattered more widely than their uncertainties, the normalised weighted $\chi^{2}$ turning out at 5.4 with slopes and 17.5 without. Thus, the uncertainties shown in the figure can be scaled up with a factor of 2 and 4, respectively. Since slopes will correlate with asymmetric tidal arcs, the $S_{c}$ 's are expected to 
scatter more than in the slopeless setting; with slopes included, however, also the uncertainties become much larger, leading to the lower $\chi^{2}$, in case you wondered. Without slopes estimated, a bias due to their presence cannot be excluded. Yet, in the ensemble average the weighted means come close to the values of the multi-campaign joint adjustment prepared in two variants, with an adjusted SCG-drift curve, alternately inforcing the drift as provided from the SCG extended analysis (the topic of the next section). The latter variant's $S$ turned out to be compatible with the campaign in Feb. 2015 when the quantum gravimeter GAIN was operating for three weeks at OSO (Freier et al., 2016). GAIN produced the scale factor with a precision of 0.027 percent.

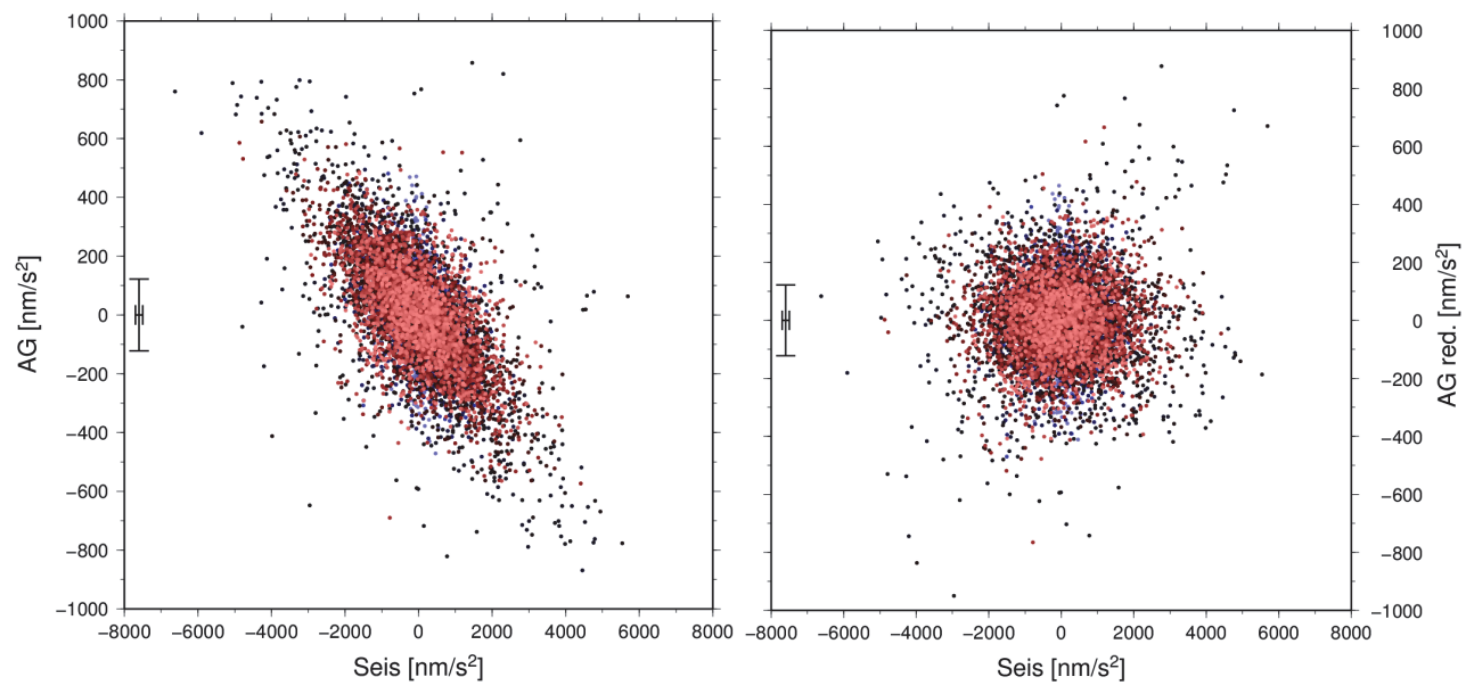

Fig. 2. Mitigating AG drop noise with the seismometer. The RMS could be reduced to a more normal level. The data set is the same as in Fig. 1, but with a clock offset adjustment for each segment.

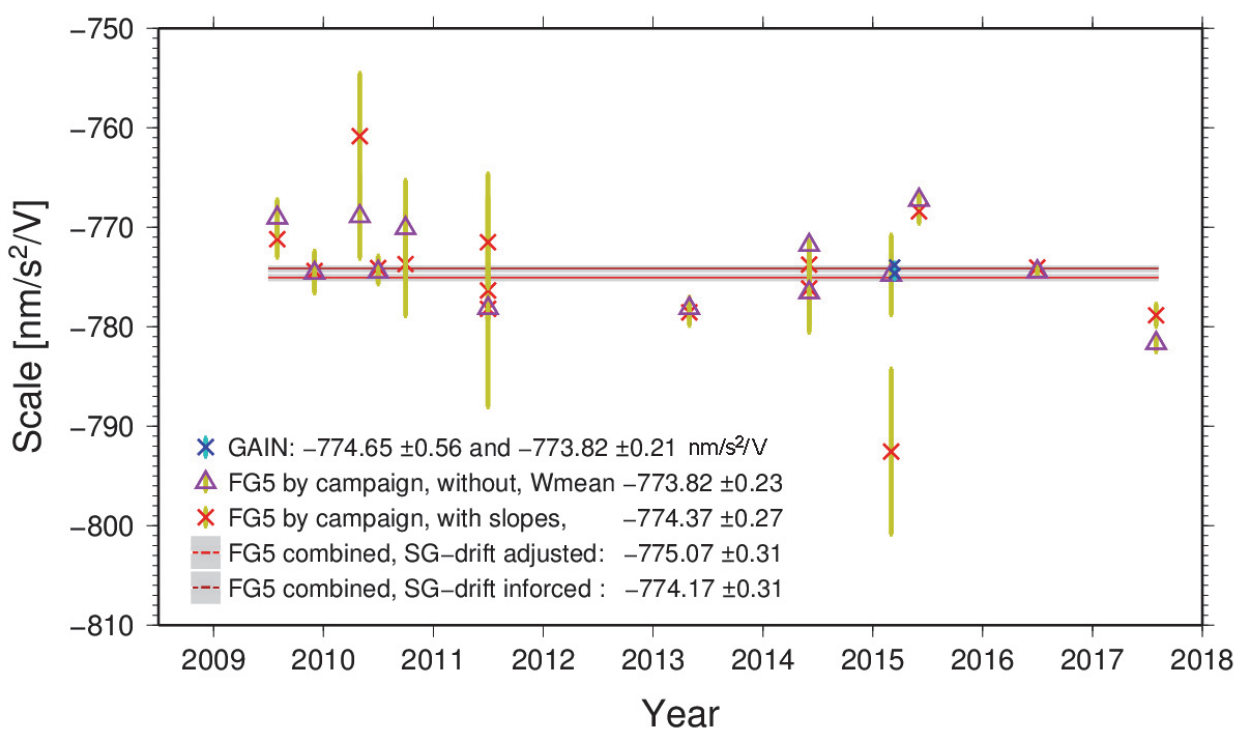

Fig. 3. Calibration of the SCG with AGs, in single campaigns, in the multi-campaign adjustment, and using GAIN, the quantum gravimeter. In single campaigns two cases are distinguished, estimating setup slopes or not; the scale factors printed out are the weighted means. Slopes have been included in the multi-campaign adjustments; there, two cases are distinguished, solving an admittance parameter for the SCG-drift curve or subtracting it as determined from SCG data alone. 
The central part of the exercise to be detailed at this point considers the extraction of the SCG's drift. The idea is to estimate the rate of change of gravity, i.e. the trend, entirely from the AG series while the SCG contributes the variations of gravity. The SCG analysis will absorb the linear part of the long-term drift along with the physical trends $\dot{g}_{p h}$ comprising GIA and more, so while an AG measures

$$
g_{A G}=g_{0}+g_{t}+g_{a}+g_{p}+g_{l}+\dot{g}_{p h} t+\varepsilon_{d}
$$

(where $t$ denotes time and $\varepsilon d$ drop noise) the SCG will report

$$
g_{S C G}=g_{t}+g_{a}+g_{p}+g_{l}+d+\varepsilon_{S C G}
$$

where the subscripts $t, a, p$ and $l$ respectively denote tides, atmosphere, polar motion, and local gravity variations, and $d$ is an apparent drift,

$$
d=d_{i}+\dot{g}_{p h} t
$$

with $\dot{d}_{i}$ the instrument's contribution proper. Then

$$
g_{A G}-\left(g_{S C G}-d\right)=g_{0}+\dot{g}_{p h} t-\dot{d}_{b} t+\varepsilon_{S}
$$

where $\varepsilon_{s} \simeq \varepsilon_{d}$ owing to low-pass filtering of the SCG data, and $\dot{d}_{b}$ anticipates a systematic bias; we'll come to it soon. The art in here is to determine $d$ such that the influence of systematic errors in its linear trend $\dot{d}=$ const. separating it from the non-linear part $\hat{d}$

$$
d=\hat{d}+\dot{d} t
$$

remains well below the uncertainty of $\dot{g}_{p h}$, which depends only on the AG measurements. Equation (4) is a radical step into hitherto unexplored terrain: in relying on the drift determination on the basis of SCG data and a priori quasi-deterministic signal model; positing the left-hand side of (4) to completely reduce all temporal non-linear variation of $g_{A G}$ except the drop noise; and not least using the original drop measurements, the method formulates another alternative to those presented in Van Camp et al. (2013).

Of particular importance in the determination of $d$ in (2) are two categories of slope biases, first the composition of the signals that represent the instrumental source of $d$, and second the linear functions of time in each of the signals representing physical contributions. The drift is solved in a least-squares adjustment, an extended tide analysis, where for instance the site-specific $g$ l is composed partly of predictions and partly of proxy-observations. Predictions are available for tides (Tamura, 1987), the atmosphere (Atmacs; Klügel and Wziontek, 2009), polar motion (Wahr, 1985, with IERS data), hydrological and nontidal ocean loading effects (ERAin and ECCO1 from EOST Loading Service; Boy et al., 2009); and tide gauge readings (reduced to represent bot- 
tom pressure are purposed to admit mass-related effects due to sea level anomalies only).

Concerning effects from groundwater, a problem plaguing many gravity stations, countermeasures were taken already in the building phase of the Onsala site, but also the geological setting on crystalline bedrock proved advantageous. An account of this can be read in (Scherneck and Rajner, 2019, A.3).

Data processing and analysis employs bespoke software, eclectic in adopting pieces from well-publicised code like an early version of ETERNA (Wenzel, 1996, v. 3.40) for computing luni-solar tides. The program called urtapt can simultaneously adjust a wide range of time series carrying gravity effects, and it interacts with crossspectral analysis constructing Wiener filters that help to admit signal features with spectral selectivity. Decorrelation of the input to meet the requirements of least-squares regression is carried out with prediction error filters (PEF) using the maximum entropy algorithm of Burg (1972). With emphasis on the long time scale the short length of PEF's has the advantage that they increase data gaps only benignly, and urtapt can cope with this; in new ETERNA ET34-X-V61-A (Schüller, 2015) such breaks necessitate segmentation or interpolation (besides that, lack of open source code impeded installation on a Linux platform). Another advantage of PEF's is their stemming from a finite Markov-chain concept, i.e. the random process is assumed stationary (including a return to a stable mean) not withstanding a quasi-deterministic slope, which it lets pass albeit with attenuation. We contend, in accord with Van Camp et al. (2010) (discussing groundwater) that a stable mean for gravity variations is a realistic assumption, atmospheric effects being the most conspicuous random component, prediction errors scaling with signal amplitude, and atmospheric mass being largely conserved as evidenced by the standard global mean surface pressure of $1013.25 \mathrm{hPa}$. Stability of this kind would not hold for e.g. horizontal displacement, which could develop unbounded (as in plate tectonic motion), thereof the approach of e.g. Williams (2003) assuming non-stationary noise (infinite Markov chain) in GNSS position determination. A Monte-Carlo simulation shows the feasibility of PEF's to represent the noise in gravity (Fig. 4). More detail is provided in (Scherneck and Rajner, 2019, A1) concerning urtapt and in (Scherneck and Rajner, 2019, A2) concerning data management and building the model matrix for regression.

None of the predictions are taken as corrections but with one exception, the nodal tide. Its $18.6 \mathrm{yr}$ period does not allow an unbiased adjustment. For all other signals the slope biasses are computed and summed up to a corresponding slope bias in

$$
\dot{d}_{b}=-\sum_{k} \dot{g}_{k} p_{k}
$$

where enumerates the set of tides, polar motion etc., and $p_{k}$ are the adjustment coefficients. For the nodal tide we assume a tidal delta factor taken from Dehant et al. (1999). Each of these series are adjusted and obtain a regression coefficient owing to their wideband character. The coefficient multiplied with the slope's $p_{k}$ is indicative of a trend 
component in $g_{p h}$. The SCG, owing to continuity of operation, thus enables us to present quantified hypotheses on trend contributions, while an effort of disentanglement with AGs' sparse campaign schedules only would appear utmost elusive.

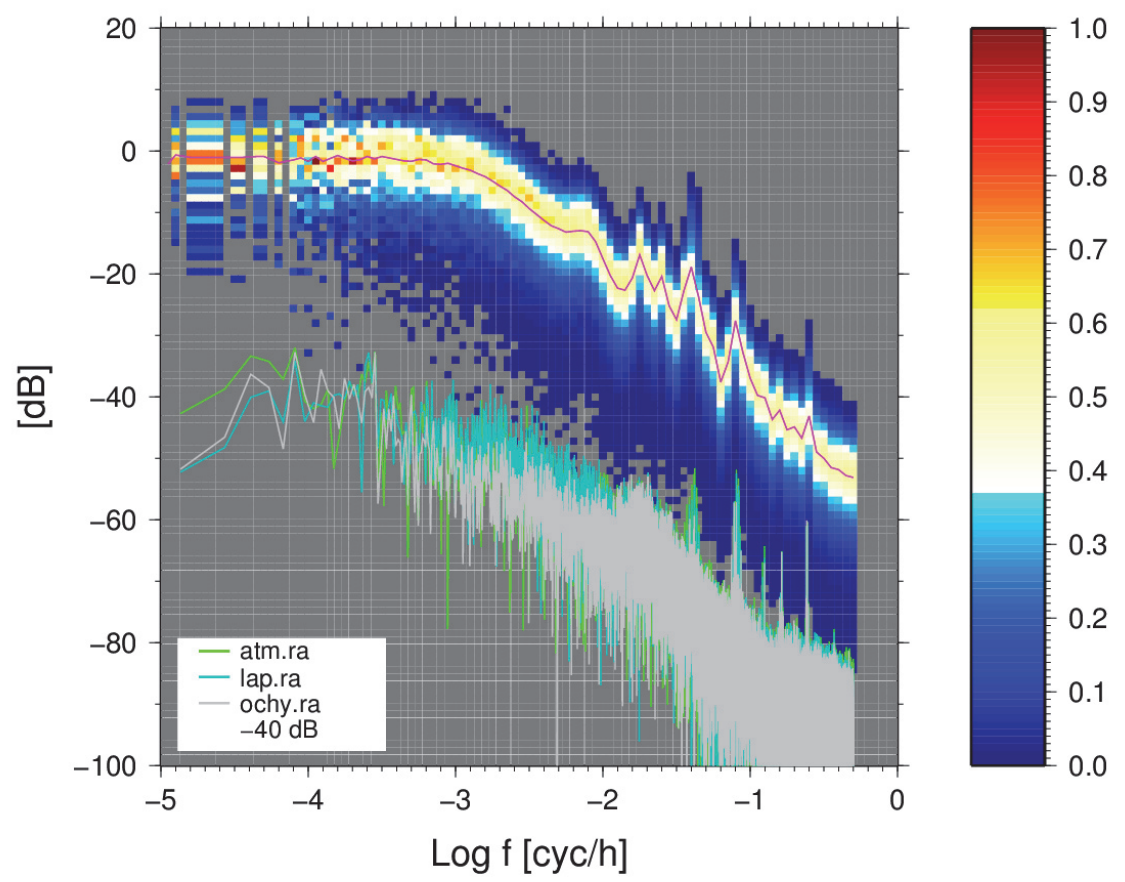

Fig. 4. Monte-Carlo test of noise-colour modelling of SCG's residual using prediction-error filters (PEF) compatible with an extended Gauss-Markov process. One hundred noise innovations and a PEF length of 40 produced the rainbow-coloured histogram, colour designating the fraction of peak occurrence (enhanced by the red line). The periodograms, drawn at a $-40 \mathrm{~dB}$ offset for readability, show the actual noise spectrum. Three analysis variants (see section 5.1) have been used the time series of which can be seen in Fig. 6, lap - local air pressure; atm - Atmacs atmosphere; ochy - nontidal ocean loading, hydrology, and Atmacs atmosphere; a bottom pressure proxy from sea level is always included.

The instrumental part contains in our case two exponentials the decay parameter of which must be solved (simultaneous with the linear drift parameters) in a non-linear least-squares fit. This step, interlaced with the linear fit, is re-iterated into selfconsistency.

The residual of the AG-SCG multi-campaign analysis also shown in Fig. 6 suggests limitations of AG campaigns to improve the SCG's drift model; both owing to internal variance on the order of 10-20 if not occasionally $50 \mathrm{~nm} / \mathrm{s}^{2}$, and to external variance in conflation, order of $50 \mathrm{~nm} / \mathrm{s}^{2}$. Notably, the international comparison campaigns and the degrees of equivalence (DoE) they attach to an AG each time outnumber the branches of the SCG drift curve.

\subsection{AG multi-campaign analysis with SCG backing}

The strategy we employ to fit a long-term trend to the reduced AG-readings at the drop level. Presently the data set comprises nearly 200,000 ordinates. Adjustment is with respect to monument AA, meter FG5-233 in North-orientation. Bias parameters are estimated for the ties to the other platforms, one for the other instrument (FG5-220), and one for each instrument's orientation. 
A scale factor for the SCG can be estimated separately in each campaign. Results may easily scatter wider than their uncertainties, which might lead to misinterpretation. Crossley et al. (2018) carefully revisited the problem. Indeed, in our case the scatter was profound. However, already a simple ensemble average came close to the value of the multi-campaign joint adjustment, an approach not considered by Crossley et al. (2018). That value turned out to be compatible within its uncertainty with the campaign in Feb. 2015 when the quantum gravimeter GAIN was operating for three weeks at OSO (Freier et al., 2016). GAIN produced the scale factor with a precision of 0.027 percent. Variants of the multi-campaign analysis may either use a fixed scale factor or adjust it.

For every project a centered slope is estimated to capture trends e.g. due to sagging verticalisation. The driftfreed SCG data is purified for microseismic noise, and multiplied with the GAIN-derived scale factor. Alternately, admittances for SCG and drift can be estimated, mostly for a test on consistency, anticipating fingerprints due to remaining offsets between some of the AG-campaigns. For initial estimates we use DoE from the international comparison campaigns (Jiang et al., 2012; Francis et al., 2013, 2015; Pálinkáš et al., 2017). In this report we limit the aim to find the range of variations of the estimated long-term SCG rate and its uncertainty while available options in the setting are enacted.

\section{Discussion}

The formal 1- $\sigma$ uncertainty for a gravity rate in the multi-campaign adjustment turns out at $\pm 0.4 \mathrm{~nm} / \mathrm{s}^{2} / \mathrm{yr}$ with a normalized $\chi^{2}$ of only 0.23 . Thus, the weights given by the drop uncertainty are generally two times too low. Giving more weight to achieve a $\chi^{2}$ closer to unity, the analysis could determine a multi-year average rate at $0.13 \mathrm{~nm} / \mathrm{s}^{2} / \mathrm{yr}$. In 13 of the 77 projects the estimated rate of the AG drop series exceeds its uncertainty by more than a factor of two. The difference from the start to the end of those is regularly above $20 \mathrm{~nm} / \mathrm{s}^{2}$ with both positive and negative slopes.

On the SCG side of the problem, for the period of the urtapt analysis, Jun. 15, 2009 until Dec. 31, 2017, a slope bias due to the node tide would remain below, say, $0.5 \mathrm{~nm} / \mathrm{s}^{2} / \mathrm{yr}$ if the tidal delta factor, prescribed as 1.1647 , is accurate to within \pm 0.008 . Thus, we identify the nodal tide prediction's accuracy as the most critical parameter in our analysis.

The slope bias in the tides-and-more analysis adds up to -0.500 with the leading contributions from ERAin hydrology's non-local part $(-0.26 \pm 0.03)$, polar motion inphase and cross-phase $(0.273 \pm 0.005$ resp. -0.125$)$, and from the long-period part of the tide gauge $(-0.073 \pm 0.015)$, all values in $\mathrm{nm} / \mathrm{s}^{2} / \mathrm{yr}$.

Still being in the phase of testing a hypothesis, this approach adopts a route of attack that differs in a number of aspects from what most of the literature on the subject offers. The multi-campaign adjustment inspired by Wziontek et al. (2009) bypasses the conflation of individual AG campaigns' errors. Instead of weighing together SCG scale factors from a set of campaigns (Crossley et al., 2018), biased as they may be, we use all campaigns simultaneously, every single drop measurement, also enabling the intro- 
duction of adjustable slopes in each setup. As of current, the slopes are centered at midproject time; if we would find a diagnostic parameter correlating with the signs found, we could infer a general, systematic trait and anchor them at project start. In that case, not only a bias and excess variance in the SCG scale factor but also a bias in the AG offsets between projects (and campaigns) could be mitigated. Here we can at least reduce the former bias.

An examination of results per project within a campaign shows variation of weighted means between 3 and $25 \mathrm{~nm} / \mathrm{s}^{2}$, i.e. regularly factor of ten larger than the weighted means' uncertainties. Anticipating that the projections of SCG rates on annual time scales are uncertain at the 1 to $2 \mathrm{~nm} / \mathrm{s}^{2}$ level, and within a campaign only limited by the scale factor known to within 0.028 percent (in units $\pm 0.3 \mathrm{~nm} / \mathrm{s}^{2}$ given the whole tidal range), the proposition to carry over DoE's to an SCG-backed station, let alone to clamp an SCG's drift component with an FG5, should raise second thoughts if not serious doubts. A test uncertainty ratio (TUR) between instruments should be greater than 4 , but if our error account is right and the SCG as reliable as this implies, the AG:SCG TUR rather appears to be 0.25 if not less.

The SCG data is low-pass filtered (and the remaining GGP filter distortion rectified) in order to suppress microseismic noise, which improves the variance ratio AG:SCG by two orders of magnitude and thus avoids a scale-factor bias (Van Camp et al., 2015), a problem that arrived with the 1-Sps data rate in the SCG models from 2008 on. Low-passing and rectifying was also applied in the GAIN campaign; comparing the latter's results with FG5 single-campaigns and the multi-campaign, GAIN will shed light on limitations in the "classic" approaches (Hinderer et al., 1991; Francis, 1997; Francis et al., 1998; Van Camp et al., 2015), for instance ignoring AG setup drift or keeping the largest source of perturbation, SCG's microseismic noise largely uncorrelated with AG's drop noise (and distorted with respect to a seismometric acceleration). As FG5 campaigns may have more objectives than to calibrate an SCG, interests we largely share, swap of orientations and platforms detrimental to that aim but necessary e.g. to pursue interest in GIA, the ideal calibration campaign might not be feasible to realise; nor would it be a sine-qua-non, if our approach indeed succeeds to compose the steps of processing synergetically and shirk unnecessary compromises.

\subsection{Tidal analysis}

Here we compare results of urtapt with commonly used software for tidal analysis, namely ETERNA3.40 (Wenzel, 1996) and Baytap08 (Agnew, 2018; Tamura et al., 1991), a Bayesian approach. The summary is given in Table 3. The percentage differences of estimated tidal factors are usually below the one-percent level. The notable exception is $S_{a}$, which we content arises from the adjustment of the environmental time series that had been deprived of seasonal effects to increase the degree of freedom for harmonic versus non-harmonic components in each of them. An interpretation of the $S a$ phasor is shown in Fig. 5. 
Table 3. Comparison of tide solutions using urtapt, ETERNA3.40, and baytap08. Shown are tidal coefficients $\delta$ and $\kappa$, the latter denoting phase lag in degrees. They include the ocean loading effect.

\begin{tabular}{|c|c|c|c|c|c|c|c|c|c|c|}
\hline \multirow[t]{2}{*}{ Tide } & \multirow{2}{*}{$\begin{array}{l}\text { Freq. } \\
\text { [cpd] }\end{array}$} & \multirow{2}{*}{$\begin{array}{l}\text { Amp. } \\
{\left[\mathrm{nm} / \mathrm{s}^{2}\right]}\end{array}$} & \multirow{2}{*}{$\begin{array}{c}\text { Theo } \\
\delta\end{array}$} & \multicolumn{2}{|c|}{ urtapt } & \multicolumn{2}{|c|}{ ETERNA } & \multicolumn{2}{|c|}{ baytap08 } & \multirow{2}{*}{$\begin{array}{c}\text { urtapt }^{-} \\
\text {-ETERNA[\%] }\end{array}$} \\
\hline & & & & $\delta$ & $\kappa$ & $\delta$ & $\kappa$ & $\delta$ & $\kappa$ & \\
\hline$S_{a}$ & 0.002738 & 5.3700 & 1.1623 & 2.0641 & 2.0641 & 1.7926 & -12.29 & & & 15.1 \\
\hline$S_{s a}$ & 0.005476 & 33.8220 & 1.1623 & 1.0819 & -4.08 & 1.0901 & -1.13 & & & -0.8 \\
\hline$M_{m}$ & 0.036292 & 38.4010 & 1.1623 & 1.1345 & -0.43 & 1.1179 & 1.19 & & & 1.5 \\
\hline$M_{f}$ & 0.073202 & 72.6840 & 1.1623 & 1.1530 & 0.18 & 1.1271 & 0.41 & & & 2.3 \\
\hline$M_{t}$ & 0.109494 & 13.9160 & 1.1623 & 1.1479 & 0.24 & 1.1272 & 0.12 & & & 1.8 \\
\hline$M_{q}$ & 0.145785 & 2.2220 & 1.1623 & 1.1516 & -0.31 & 1.1088 & 2.27 & & & 3.9 \\
\hline$Q_{1}$ & 0.893244 & 53.9450 & 1.1523 & 1.1400 & -0.34 & 1.1354 & -0.31 & 1.0829 & 1.03 & 0.4 \\
\hline$O_{1}$ & 0.929536 & 281.6990 & 1.1520 & 1.1437 & 0.09 & 1.1391 & 0.13 & 1.1091 & -0.17 & 0.4 \\
\hline$M_{1}$ & 0.966137 & 22.1430 & 1.0728 & 1.1491 & 0.26 & 1.0827 & 0.72 & 1.1363 & 8.60 & 6.1 \\
\hline$P_{1}$ & 0.997262 & 130.4630 & 1.1465 & 1.1515 & 0.06 & 1.1453 & 0.10 & 1.1124 & 0.03 & 0.5 \\
\hline$S_{1}$ & 1.000000 & 3.0750 & 1.1431 & 1.0473 & -2.21 & 1.0874 & 1.46 & 0.7124 & 163.96 & -3.7 \\
\hline$K_{1}$ & 1.002738 & 389.5200 & 1.1310 & 1.1368 & 0.10 & 1.1317 & 0.14 & 1.1021 & 0.22 & 0.4 \\
\hline$\psi_{1}$ & 1.005476 & 3.3450 & 1.2367 & 1.2811 & 0.37 & 1.2620 & 1.06 & 0.6978 & 106.36 & 1.5 \\
\hline$\phi_{1}$ & 1.008214 & 5.7150 & 1.1670 & 1.1771 & -0.34 & 1.1726 & -0.01 & 1.5249 & 4.13 & 0.4 \\
\hline$J_{1}$ & 1.039030 & 22.1900 & 1.1541 & 1.1551 & -0.17 & 1.1526 & -0.11 & 1.0326 & -2.21 & 0.2 \\
\hline$O O_{1}$ & 1.075940 & 12.1320 & 1.1533 & 1.1468 & 0.05 & 1.1459 & -0.01 & 1.1280 & -3.61 & 0.1 \\
\hline $3 N_{2}$ & 1.823399 & 0.2650 & 1.1566 & 1.1032 & 0.59 & 1.0980 & 2.80 & & & 0.5 \\
\hline$\varepsilon_{2}$ & 1.828256 & 1.6030 & 1.1566 & 1.1168 & -0.47 & 1.1150 & -0.74 & 0.8633 & -1.94 & 0.2 \\
\hline $2 N_{2}$ & 1.859690 & 5.4970 & 1.1566 & 1.1371 & 2.48 & 1.1508 & 2.62 & 1.1804 & 1.46 & -1.2 \\
\hline$\mu_{2}$ & 1.864547 & 6.6340 & 1.1566 & 1.1231 & 0.90 & 1.1114 & 0.89 & 1.0735 & -2.66 & 1.1 \\
\hline$N_{2}$ & 1.895982 & 41.5430 & 1.1566 & 1.1729 & 2.01 & 1.1696 & 2.11 & 1.1160 & 2.49 & 0.3 \\
\hline$\alpha_{2}$ & 1.929536 & 0.7460 & 1.1566 & 1.2327 & -8.57 & 1.1769 & -9.05 & 3.5416 & 34.34 & 4.7 \\
\hline$M_{2}$ & 1.932274 & 216.9680 & 1.1566 & 1.1827 & 1.21 & 1.1787 & 1.30 & 1.1504 & 1.20 & 0.3 \\
\hline$L_{2}$ & 1.968565 & 6.1320 & 1.1566 & 1.1817 & -0.49 & 1.1750 & -0.43 & 1.2498 & 1.96 & 0.6 \\
\hline$T_{2}$ & 1.997262 & 5.8990 & 1.1566 & 1.1751 & 0.57 & 1.1754 & 0.65 & 0.9174 & -1.85 & -0.0 \\
\hline$S_{2}$ & 2.000000 & 100.9360 & 1.1566 & 1.1744 & 0.28 & 1.1709 & 0.34 & 1.1524 & 1.06 & 0.3 \\
\hline$R_{2}$ & 2.002738 & 0.8440 & 1.1566 & 1.1687 & 0.03 & 1.1683 & -0.27 & & & 0.0 \\
\hline$K_{2}$ & 2.005476 & 27.4350 & 1.1566 & 1.1762 & 0.42 & 1.1723 & 0.51 & 1.1807 & 0.02 & 0.3 \\
\hline$\eta_{2}$ & 2.041767 & 1.5340 & 1.1566 & 1.1749 & 0.24 & 1.1707 & 0.23 & & & 0.4 \\
\hline$M_{3}$ & 2.898410 & 2.2880 & 1.0684 & 1.0707 & 1.13 & 1.0675 & 1.27 & 1.0330 & 2.79 & 0.3 \\
\hline$M_{4}$ & 3.864547 & 0.0220 & 1.0346 & 5.7599 & -163.14 & 5.9791 & -167.00 & & & -3.7 \\
\hline
\end{tabular}

In the diurnal and semidiurnal bands the agreement between the different solutions is clear. The deviations from the theoretical values of Dehant et al. (1999) are larger owing to the ocean tidal loading effects that were kept unreduced. Also, note the very strong resonant effect for the nonlinear constituent $M_{4}$ revealed both by urtapt and ETERNA).

The regression that includes a range of environmental series and drift model segments (the extended set) produces the estimated drift shown in Fig. 6 along with the residual. In the same figure we also show the results from simpler sets in the regression (local barometer instead of Atmacs; neither ERAin nor ECCO1). The differences of the mean $\dot{d}$ between these three versions are large, order of $5 \mathrm{~nm} / \mathrm{s}^{2} / \mathrm{yr}$, and are attributable to the inclusion of ERAin and ECCO1, vindicating our interpretation of the contributors to the observed $S_{a}$ tide. In order to find out how the drift's $\dot{d}$ depends on the noise (i.e. signal unaccounted for in the fit) we used a Monte-Carlo analysis (MC) with the ex- 
tended set. Its stochastics were noise-coloured with a prediction-error filter representative of the residual. The finding suggests a standard deviation of $0.2 \mathrm{~nm} / \mathrm{s}^{2} / \mathrm{yr}$ for $\dot{d}$. In the most recent drift branch starting Sep. 12, 2016, the offset uncertainty from MC is $0.6 \mathrm{~nm} / \mathrm{s}^{2}$.

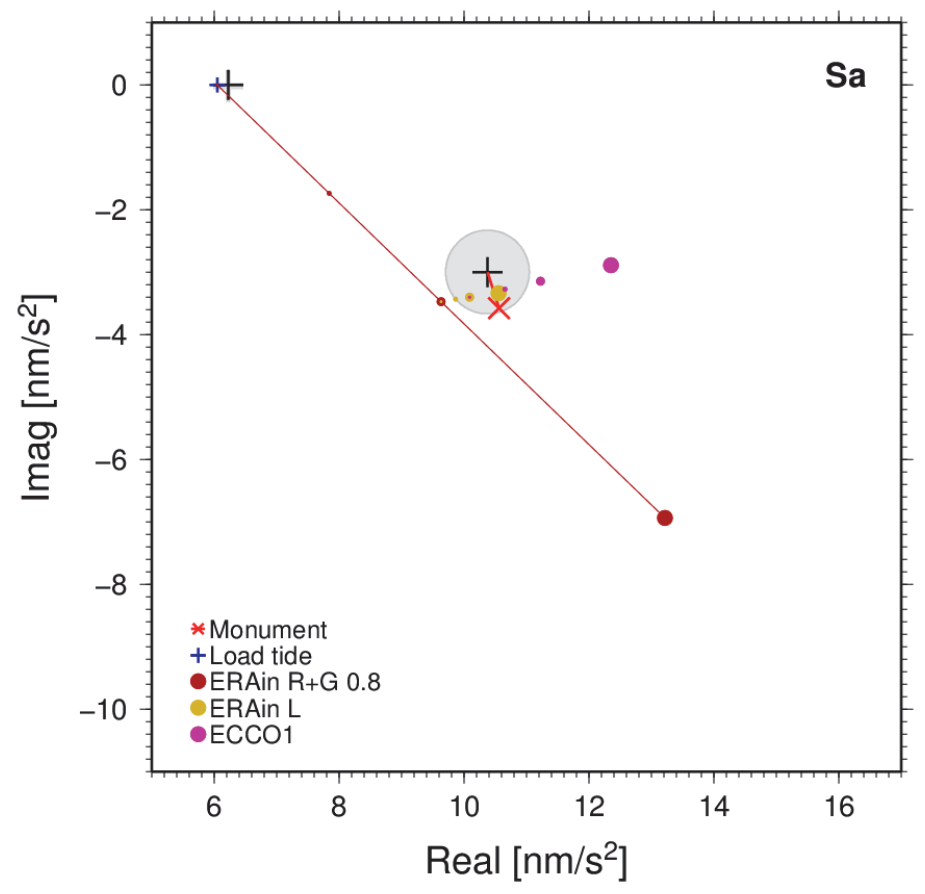

Fig. 5. Phasor diagram for the annual tide $S_{a}$. Fraught with seasonal environmental impacts as it is, a breakdown into constituents cannot but turn out ambiguous when a single spectral line is dissected. The observed phasor is shown as a black cross surrounded by its ellipse of uncertainty $(1-\sigma)$ in gray. The solid earth tide is represented by the black cross at the top left; a purely elastic earth was assumed. For ocean tide loading we surmised an equilibrium tide, after its tiny contribution the result is shown in blue. The height of the SCG monument is continuously measured with an Invar-rod. The inferred gravity change, subtracted from the observation, is shown in light-red. The environmental effects predicted from ERAin and ECCO1 are good candidates to explain the large effect observed. The diagram shows ERAin split into regional and global parts $(\mathrm{R}+\mathrm{G}$, dark-red) and a local part (L, yellow), and ECCO1 (purple), each as straight lines ending at the full effect and beads along the lines at one-half and one-quarter We construe a plausible a fit with ERAin $\mathrm{R}+\mathrm{G}$ at half of its predicted effect, while ECCO1 and ERAin L can be traded against each other as both have similar phase angles, while the near orthogonal conjuncture of ERAin $\mathrm{R}+\mathrm{G}$ and ECCO1 might suggest that both overpredict their impacts - unless we have overlooked the most powerful contributor of annually varying gravity.

The residual of the extended analysis still seems to exhibit features worth contemplation. At the OSO site, exposed to westerly weather patterns, wind-driven sea level variations in suit, a gravity effect is measurable hours before the barometer and the load-effective mass on OSO's side of Kattegat react. They are not possible to capture with a time-independent admittance model. Although Atmacs is designed to represent the 3-D effect of atmospheric density structure varying with time, a non-static response of the sea to surface pressure implies an explicit time dependency and thence an account of the hydrodynamics at play. A rough calculation suggests, if the long-period features of the gravity residual has an RMS of $5 \mathrm{~nm} / \mathrm{s}^{2}$ and air pressure one of $10 \mathrm{hPa}$ admitted with $-3.5 \mathrm{~nm} / \mathrm{s}^{2} / \mathrm{hPa}$, the atmospheric model mispredicts at a 15 percent level were it the only one responsible. Blaming it on ERAin or ECCO1 would suggest much higher, 
first-order error levels. Either of these assertions may appear stark, not least noting that the admittance coefficients are rather well determined; relative uncertainties are for ERAin 0.06, for ECCO1 0.028, for the bottom pressure proxy 0.02, and for Atmacsattraction 0.004 (the latter's loading effect is admitted at 0.6 where it should be unity $\pm 0.035)$.

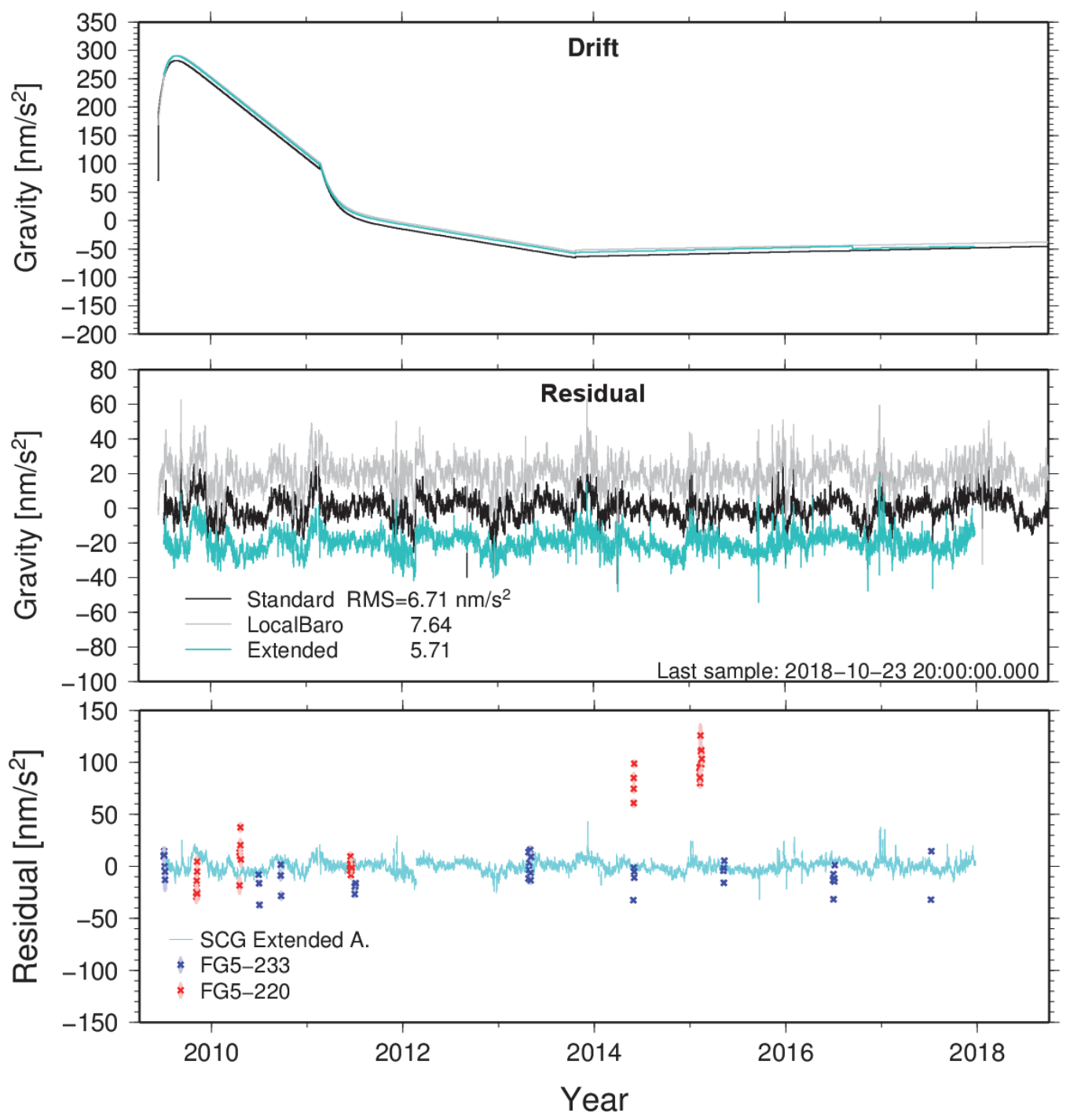

Fig. 6. The drift (top diagram) and the least-squares residuals (mid) from the SCG data analysis in three variants. "Standard" implies the use of Atmacs atmosphere, in "Extended" the effects of hydrology and non-tidal ocean loading are added using gravity predictions from ERAin and ECCO1 available from the IGETS loading service. "LocalBaro" uses none of these but the local barometer as a proxy for atmospheric perturbations. A mass anomaly proxy for near-by Kattegat is always included using a reduced sea-level record composed of the tide gauge at Ringhals and, from 2012 on, OSO's own. The breaks in the drift signal coincide with the repair of a control card in spring 2011 and a dodgy cold-head swap in autumn 2013. Another one in mid 2016 has been introduced provisionally; it's amplitude was found to decrease as the record length grew; it's still solved for in the extended analysis. In the standard residual you might notice fingerprints of a wet summer in 2016 and a dry one in 2018. The excursion in mid 2016 appears smaller in the extended analysis at least. In the broad picture the anomalies visible in the residuals do take part in the reduction of the AG data as do any of the admitted models and proxies, reaching beyond the capabilities of reduction enacted by the Micro-g software. In the bottom diagram the residuals of the AGmulti-campaign analysis averaged over each project are shown. Conspicuously, two campaigns with the FG5X-220, one in 2014 and one 2015, stick out. Their DoE's were only different by $6 \mathrm{~nm} / \mathrm{s}^{2}$, up in 2015. Their internal spreads are on the order of $50 \mathrm{~nm} / \mathrm{s}^{2}$. 
Comparing the residuals of the multi-campaign adjustment with the SCG residual (Fig. 6) we notice that the spread of the former within the same campaign regularly exceeds the range of the latter. There is little possibility to regularize the behaviour. However, clear signs of outliers in singular projects and even an outlier campaign (like the one with the FG5-220 in 2014, simultaneously with the FG5-233, and another in 2015, may lead to reevaluation of the campaign or the DoE that was applied. Another aspect that comes to the fore in Fig. 6 is the impact the FG5-220 campaigns in 2014 and 2015 exert on the variant of the super-campaign analysis where a scale for the SCG-drift curve is estimated, a change of $6 \%$ much owed to these two campaigns.

Another piece of evidence in favour of applying the SCG drift curve at unity scale is the higher consistency found in the discussion of the scale factor. In the Monte-Carlo test of the SCG analysis confidence intervals for the drift parameters are found at (Scherneck and Rajner, 2019, A2.5) $\pm 0.5-1.5 \mathrm{~nm} / \mathrm{s}^{2} / \mathrm{yr}$ and $1 \mathrm{~nm} / \mathrm{s}^{2}$ for slopes and offsets, respectively. A mean slope straddling the entire time span shows an uncertainty of $\pm 0.25 \mathrm{~nm} / \mathrm{s}^{2} / \mathrm{yr}$. This, together with the residual RMS of less than $5 \mathrm{~nm} / \mathrm{s}^{2}$ suggests that the SCG data is sufficiently coherent on the multi-year scale to reduce gravity variations observed in AG campaigns, which will enhance visibility of instrumental problems in the latter.

\section{$6 \quad$ Conclusions}

We have laid out a route to simultaneously adjust the whole series of absolute gravity campaigns at Onsala Space Observatory, using AG data at the drop level. Instead of a priori models for gravity's temporal variations, synchronous samples from the superconducting gravimeter OSG-054 (SCG) are used in the reduction. We propose that the accomplished separation of instrumental contributions from long-term gravity change is well-constrained. One of the advantages is the detectability of setup drifts in the FG5 data. In a number of cases slope significant ranges on the order of tens of nm/ $\mathrm{s}^{2}$ were found.

The residual of the SCG analysis has an RMS of less than $6 \mathrm{~nm} / \mathrm{s}^{2}$. It shows excursions of up to $\pm 20 \mathrm{~nm} / \mathrm{s}^{2}$ on annual and seasonal time scales, effects that are not fully understood, probably of regional origin in the Kattegat basin or limitations in atmospheric modelling. Prospectively, these anomalies reduce part of the gravity variations sensed by the AGs.

The drift behaviour of the SCG could be formulated parsimoniously with branches related to a few specific operational interventions, made up of simple functions (steps, linear slopes, exponential decays, and their parameters inferred from SCG data alone. The uncertainty of the long-term average is at the level of $0.25 \mathrm{~nm} / \mathrm{s}^{2} / \mathrm{yr}$.

The SCG data resolves gravity effects predicted from non-tidal ocean loading and hydrology models. The secular gravity rates these series contain have been termed rate bias in this study. However, they represent an adjusted result on the basis of which secular change in addition to glacial isostatic adjustment can be identified. 
As OSO's location is somewhat peripheral in the uplift area, demands on constraining systematic errors are higher than in the centre. We are optimistic to at least meet them at a level of $\pm 0.5 \mathrm{~nm} / \mathrm{s}^{2} / \mathrm{yr} \pm 0.6 \mathrm{~nm} / \mathrm{s}^{2}$, i.e. on the annual time scale of repeated AG campaigns a significantly lower uncertainty than the AG's degree of equivalence determined in the international campaigns. It appears worth trying to take the next step and bracket the range of estimated rates of change of gravity by exploring the degrees of freedom our approach offers. Encouraging toward this end are the low uncertainties we obtain from modelling the superconducting gravimeter's drift and the RMS of the residual.

\section{Acknowledgements}

We are indebted to a many of helping hands without this work couldn't have been considered. AG campaigns at OSO have been conducted by Andreas Engfeldt and PerAnders Olsson, Lantmäteriet; Ludger Timmen and Manuel Schilling, IfE Hannover; Christian Freier and Matthias Hauth, Humboldt University Berlin. Uppsala University's Björn Lund and Michael Schieschke have provided us access to the SNSN seismometer data, and Hossein Shomali has helped us with processing. We are grateful to Hartmut Wziontek and Thomas Klügel, BKG, for valuable advice concerning instruments and atmospheric models, and Holger Steffen, Lantmäteriet, for GIA modelling. Finally we direct our thanks to two anonymous reviewers who have helped to improve this manuscript.

\section{References}

Agnew, D.C., 2018. Baytap08 user's manual. https://escholarship.org/uc/item/4c27740c. Boy, J.-P., L. Longuevergne, F. Boudin, T. Jacob, F. Lyard, M. Llubes, N. Florsch, M.F. Esnoult, 2009. Modelling atmospheric and induced non-tidal oceanic loading contributions to surface gravity and tilt measurements. J. Geodyn., 48, 182-188. doi:10.1016/j.jog.2009.09.022.

Burg, J.P., 2018. The relationship between maximum entropy spectra and maximum likelihood spectra. Geophysics, 37:2 375-376, 1972.

Bützler, C., Drift des supraleitenden Gravimeters SG056 am BFO. Bachelor Thesis, Inst. f. Geophysik, Karlsruhe Institute of Technology. http://dx.doi.org/10.5445/IR/1000082191.

Crossley, D., M. Calvo, S. Rosat, J. Hinderer, 2018. More Thoughts on AGSG Comparisons and SG Scale Factor Determinations. Pure Appl. Geophys., https://doi.org/10.1007/s00024-018-1834-9.

Dehant, V., P. Defraigne and J.M. Wahr, 1999. Tides for a convecting Earth. J. Geophys. Res., 104, 1035-1058.

Ekman M. and J. Mäkinen, 1996. Recent postglacial rebound, gravity change and mantle flow in Fennoscandia. Geophys. J. Int. 126, 229-234. 
Francis, O., 1997. Calibration of the C021 superconducting gravimeter in Membach (Belgium) using 47 days of absolute gravity measurements. Int. Ass. Geod. Symp. 117, 212-218.

Francis, O., 1997. Calibration of the C021 Superconducting Gravimeter in Membach (Belgium) Using 47 Days of Absolute Gravity Measurements. International Association of Geodesy Symposia, Vol. 117, 212-219.

Francis, O., T.M. Niebauer, G. Sasagawa, F. Klopping, J. Gschwind, 1998. Calibration of a superconducting gravimeter by comparison with an absolute gravimeter FG5 in Boulder. Geophys. Res. Lett. 25, 1075-1078.

Francis, O., et al. 2013. The European Comparison of Absolute Gravimeters 2011 (ECAG-2011) in Walferdange, Luxembourg: results and recommendations. Metrologia, 50, 257-268.

Francis, O., et al. 2015. International comparison of absolute gravimeters (ECAG2013), CCM.G-K2 Key Comparison. Metrologia, 52, 07009, https://doiorg.proxy.lib.chalmers.se/10.1088/0026-1394/52/1A/07009.

Freier, C., M. Hauth, V. Schkolnik, B. Leykauf, M. Schilling, H. Wziontek, H.-G. Scherneck, H.-G., J. Müller and A. Peters, 2016. Mobile quantum gravity sensor with unprecedented stability. Journal of Physics: Conference Series, 723:1. https://iopscience.iop.org/article/10.1088/1742-6596/723/1/012050.

Goodkind, J. and R. Warburton, 1975. Superconductivity applied to gravimetry. IEEE Transactions on Magnetics, 11:2, 708-711.

Hinderer, J., N. Florsch, J. Mäkinen, H. Legros and J.E. Faller, 1991. On the calibration of a superconducting gravimeter using absolute gravity measurements. Geophys. J. Int. 106 (2), 491-497. doi:10.1111/j.1365-246X.1991. tb03907.x

Hinderer, J., D. Crossley and R.J Warburton, 2015. Superconducting Gravimetry, Chap. 3.04 in G. Schubert: Treatise on Geophysics, 11 Volume Set (2nd Ed.). Elsevier.

Jiang, Z. et al., 2012. The 8th International Comparison of Absolute Gravimeters 2009: the first Key Comparison (CCM.G-K1) in the field of absolute gravimetry. Metrologia, 49, 666-684. doi:10.1088/0026-1394/49/6/666.

Klügel, T. and H. Wziontek, 2009. Correcting gravimeters and tiltmeters for atmospheric mass attraction using operational weather models. In: New Challenges in Earth's Dynamics - Proceedings of the 16th International Symposium on Earth Tides. J. Geodynamics., 48:3, 204-210.

Lambeck, K., H. Rouby, A. Purcell, Y. Sun and M. Sambridge, 2014. Sea level and ice volume since the glacial maximum. PNAS, 111:43, 15296-15303. doi:10.1073/pnas.1411762111.

Meurers, B., 2012. Superconducting Gravimeter Calibration by Colocated Gravity Observations: Results from GWR C025. Int. J. Geophys., Article ID 954271.

Milne, G.A., J.X. Mitrovica, H.-G. Scherneck, J.L. Davis, J.M. Johansson, H. Koivula and M. Vermeer, 2004. Continuous GPS measurements of postglacial adjustment in Fennoscandia: 2. Modeling results. J. Geophys. Res., 109, B02412, doi:10.1029/2003JB002619. 
Olsson, P.-A., A. Engfeldt, and J. Ågren, 2016. Investigations of a suspected jump in Swedish repeated absolute gravity time series. In: J.T. Freymueller and L. Sánchez, (Eds) International Symposium on Earth and Environmental Sciences for Future Generations. International Association of Geodesy Symposia, Vol. 147. Springer, Cham.

Olsson, P.-A. K. Breili, V. Ophaug, H. Steffen, M. Bilker-Koivula, E. Nielsen, T. Oja and L. Timmen, 2019. Postglacial gravity change in Fennoscandia three decades of repeated absolute gravity observations. Geophysical Journal International, 217 (2), 1141-1156. https://doi.org/10.1093/gii/ggz054.

Olsson, P.-A., G.A. Milne, H.G. Scherneck and J. Ågren, 2015. The relation between gravity rate of change and vertical displacement in previously glaciated areas. $J$. Geodyn., 83, 76-84.

Olsson, P.-A., H.G. Scherneck and J. Ågren, 2012. Modelling of the GIA-induced surface gravity change over Fennoscandia. J. Geodyn., 61, 12-22.

Pálinkáš, V., O. Francis and M. Val'ko, M., in total 37 authors, Regional comparison of absolute gravimeters, EURAMET.M.G-K2 key comparison. Metrologia 54(1A), 07012. https://doi.org/10.1088/0026-1394/54/1A/07012 2017.

Scherneck, H.-G. and M. Rajner, 2019. Supplementary material to: Using a Superconducting Gravimeter in Support of Absolute Gravity Campaigning - A feasibility study. EarthArXiv. https://doi.org/10.31223/osf.io/yxvjc.

Schüller, K., 2015. Theoretical Basis for Earth Tide Analysis with the New ETERNA34-ANA-V4.0 Program. Mareés Terrestres, 149, 12,024-12,061.

Tamura, Y., 1987. A harmonic development of the tide- generating potential. Mareés Terrestres, 99, 6813-6855.

Tamura, Y., T. Sato, M. Ooe and M. Ishiguro, 1991. A procedure for tidal analysis with a bayesian information criterion. Geophys. J. Int., 104, 507-516.

Timmen, L., A. Engfeldt and H.-G. Scherneck, 2015. Observed secular gravity trend at Onsala station with the FG5 gravimeter from Hannover. J. Geod. Sci., 5, 18-25.

Van Camp, M., O. de Viron and R.J. Warburton, 2013. Improving the determination of the gravity rate of change by combining superconducting with absolute gravimeterdata. Computers \& Geosciences, 51, 49-55.

Van Camp, M., L. Métivier, O. de Viron, B. Meurers and S.D.P. Williams, 2010. Characterizing long-time scale hydrological effects on gravity for improved distinction of tectonic signals. J. Gephys. Res., 115, B07407, 10pp.

Van Camp, M., B. Meurers, O. de Viron and T. Forbriger, 2015. Optimized strategy for the calibration of superconducting gravimeters at the one per mille level. J. Geod. 90(1), 91-99.

Wenzel, H.-G., 1996. The nanogal software: Earth tide data processing package ETERNA 3.30. Mareés Terrestres, 124, 9425-9439.

Williams, S.D.P., 2003. The effect of coloured noise on the uncertainties of rates estimated from geodetic time series. J. Geod., 76, 483-494. 
Wziontek, H., R. Falk, H. Wilmes and P. Wolf, 2009. Precise Gravity Time Series and Instrumental Properties from Combination of Superconducting and Absolute Gravity Measurements. In: M.G. Sideris (ed.), Observing our Changing Earth, International Association of Geodesy Symposia 133, pp. 301-306, Springer-Verlag Berlin Heidelberg.

Wahr, J.M. 1985. Deformation induced by polar motion. J. Geophys. Res. Solid Earth 90, 9363-9368. doi:10.1029/JB090iB11p09363. 